\title{
Kystes osseux solitaires de la mandibule. Diagnostic, évolution et traitement
}

\section{RÉSUMÉ}

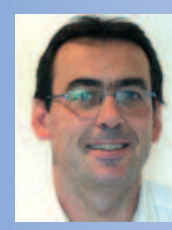

Christian MILIN

Ancien assistant hospitalo-universitaire (Paris VII)

Praticien hospitalier

Centre hospitalier Camille Guérin,

22, boulevard Aristide-Briand,

86100 Châtellerault.

La découverte d'un kyste osseux solitaire de la mandibule chez un adolescent nous permet de suivre son évolution sur quatre années. Après une revue des différentes étiopathogénies suspectées, nous présenterons les diagnostics différentiels, le traitement de ce cas clinique ainsi que deux cas de kystes osseux solitaires dont un volumineux touchant une personne âgée édentée.

kyste osseux solitaire

- diagnostic

traitement
AOS 2012;260:373-385

DOI: $10.1051 /$ aos/2012408

(C) EDP Sciences 


\section{Introduction}

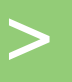

La découverte d'un kyste osseux solitaire de la mandibule dont nous pouvons suivre le développement sur quatre ans nous permet de faire le point sur les étiologies, les diagnostics différentiels et les traitements de ces pseudo-kystes.

Ces kystes sont classés dans la catégorie des tumeurs odontogènes des maxillaires par l'Organisation mondiale de la santé (1992) qui recommande le terme de kyste osseux solitaire [1], cette lésion constituant une pathologie bénigne caractérisée par une ostéolyse non tumorale, à croissance expansive.
II ne s'agit pas à proprement parler de kyste, ces lésions ne disposant pas de membranes épithéliales mais elles se présentent avec les mêmes caractéristiques clinique et radiographique (d'où la dénomination possible de pseudo-kyste).

Une grande variabilité dans la dénomination de ces lésions est retrouvée dans la littérature : kyste idiopathique, kyste essentiel, kyste osseux traumatique, kyste hémorragique, kyste hématique, cavité osseuse progressive, ostéodystrophie kystique, signant par là même les incertitudes quant à la nature et l'étiologie de ces pseudo-tumeurs.

\section{Observation clinique 1}

Monsieur J. Cor., 14 ans, sans antécédents médicaux, est envoyé par son orthodontiste pour avis et diagnostic sur une image radioclaire de la symphyse mandibulaire, découverte fortuitement au cours d'une radiographie panoramique de routine (fig. 1). II n'y a aucun signe clinique associé et seule la radiographie panoramique interpelle.

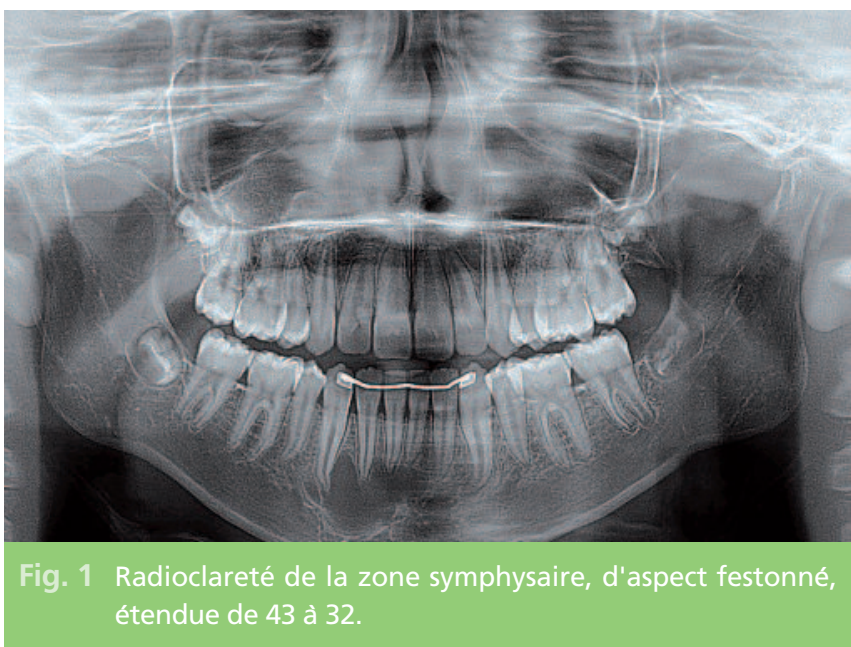

On observe une image lacunaire radioclaire, à l'apex de 43.42.41, aux limites biens définies, ceinturées de zone d'ostéoscléroses.

Les dents avoisinantes répondent positivement aux tests pulpaires, la muqueuse en regard de la lésion est normale et la palpation endobuccale est indolore.

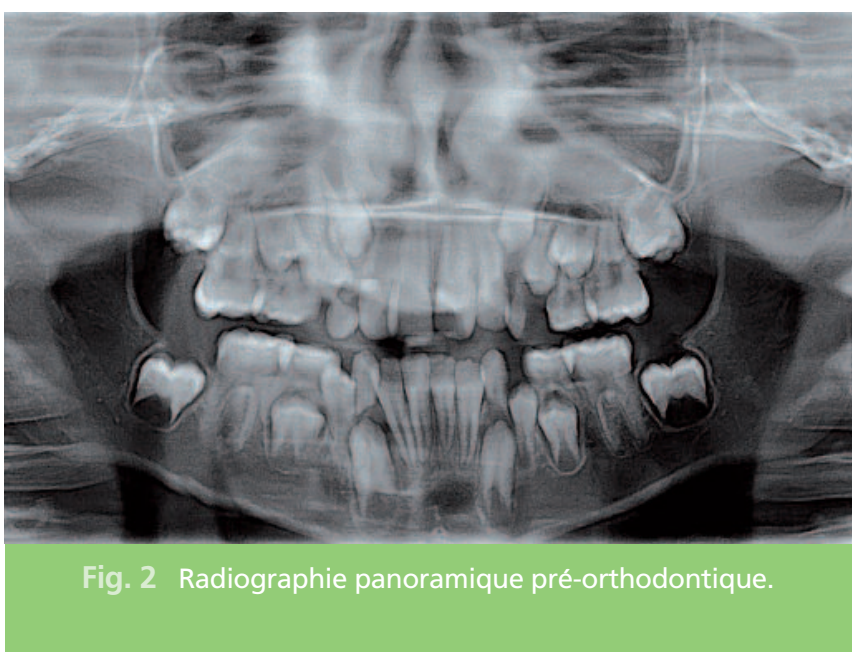


On note des antécédents d'avulsions de premières prémolaires sans difficultés particulières, effectuées sous anesthésie locale en 2008.

L'examen TDM confirme la présence d'une lacune symphysaire, aux limites bien définies, sans atteintes de corticales (fig. 5).

Nous arrivons à retrouver les anciennes radiographies panoramiques qui nous permettent de suivre l'évolution de la lésion : en 2008, aucune anomalie osseuse n'était visible (fig. 2).
À partir de 2008, une petite image lacunaire radioclaire apparaît mésialement en regard de la racine de 43, cette lacune s'accroissant progressivement en 2009, 2010 et 2011, jusqu'à impliquer les apex des dents 42, 41 et 31 avec un aspect festonné, caractéristique de ces kystes osseux solitaires (fig. 2, 3, 4).

Devant l'accroissement de la taille de la lésion, un diagnostic provisoire de kyste osseux solitaire est posé.
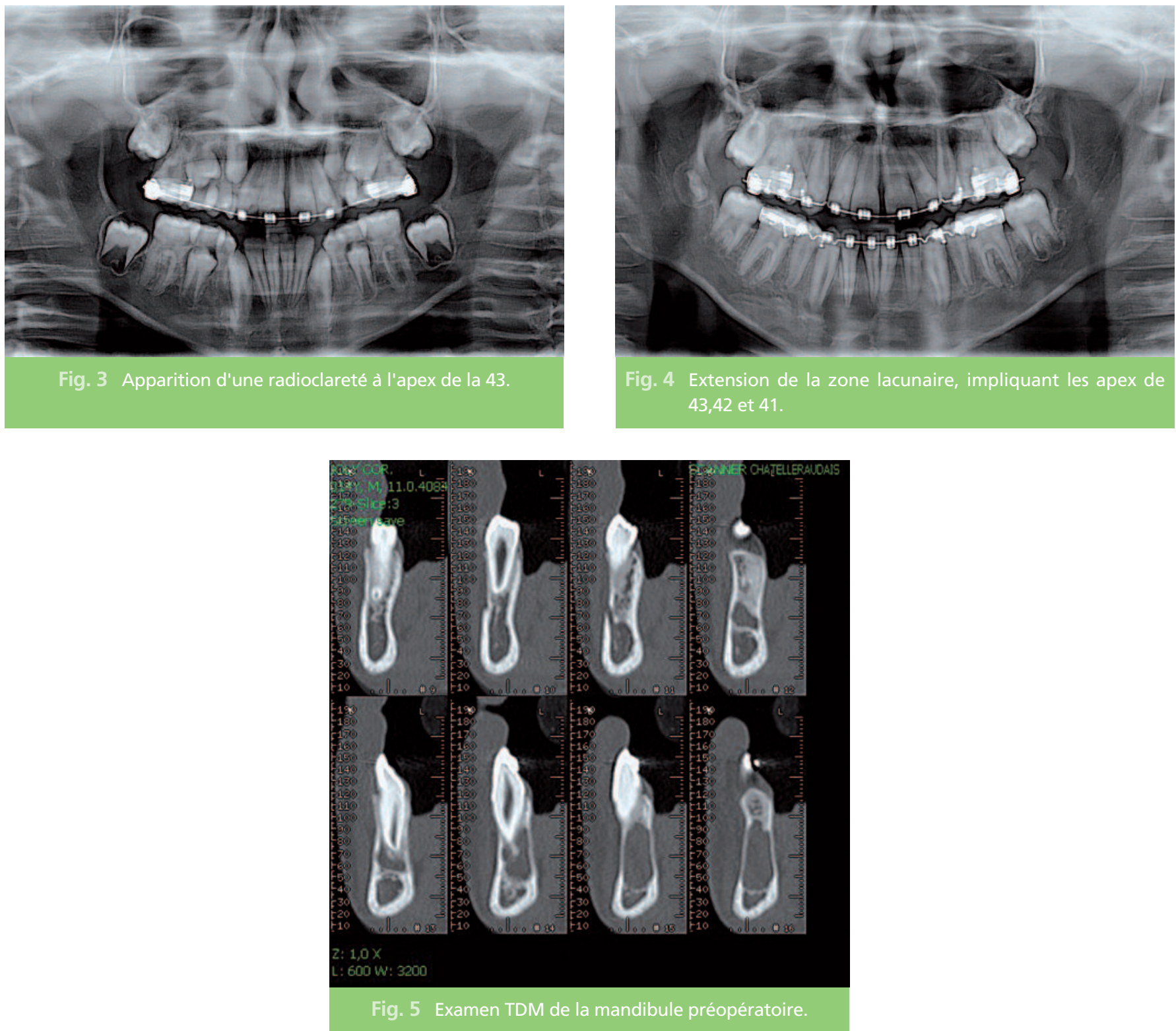
Une exploration chirurgicale est décidée, réalisée par élévation d'un lambeau mucco-périosté de 33 à 43.

L'abord chirurgical montre une modification de l'aspect du tissu osseux vestibulaire, plus opaque (fig. 6). La trépanation se réalise aisément à la fraise à os, permettant d'aboutir dans une cavité pneumatisée, recouverte d'une
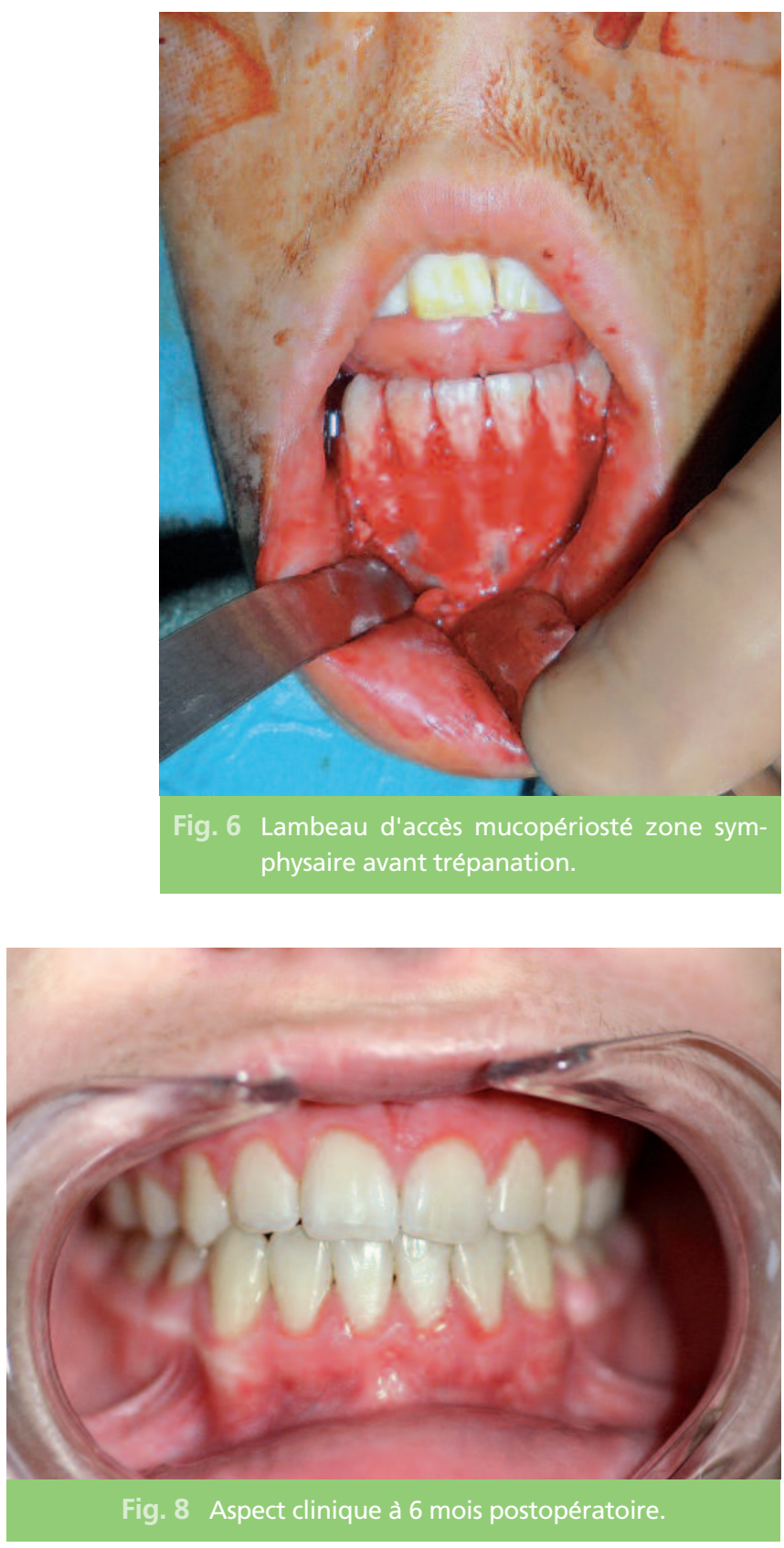

petite membrane qui s'avérera être de nature conjonctive après analyse anatomopathologique (fig. 7).

Les suites opératoires ont été simples, avec un petit œdème et de légères algies, soulagées par du paracétamol. Le contrôle clinique et radiographique à $\mathrm{J}+6$ mois montre une réossification et un aspect muqueux normal (fig. 8 et $\mathbf{9}$ ).
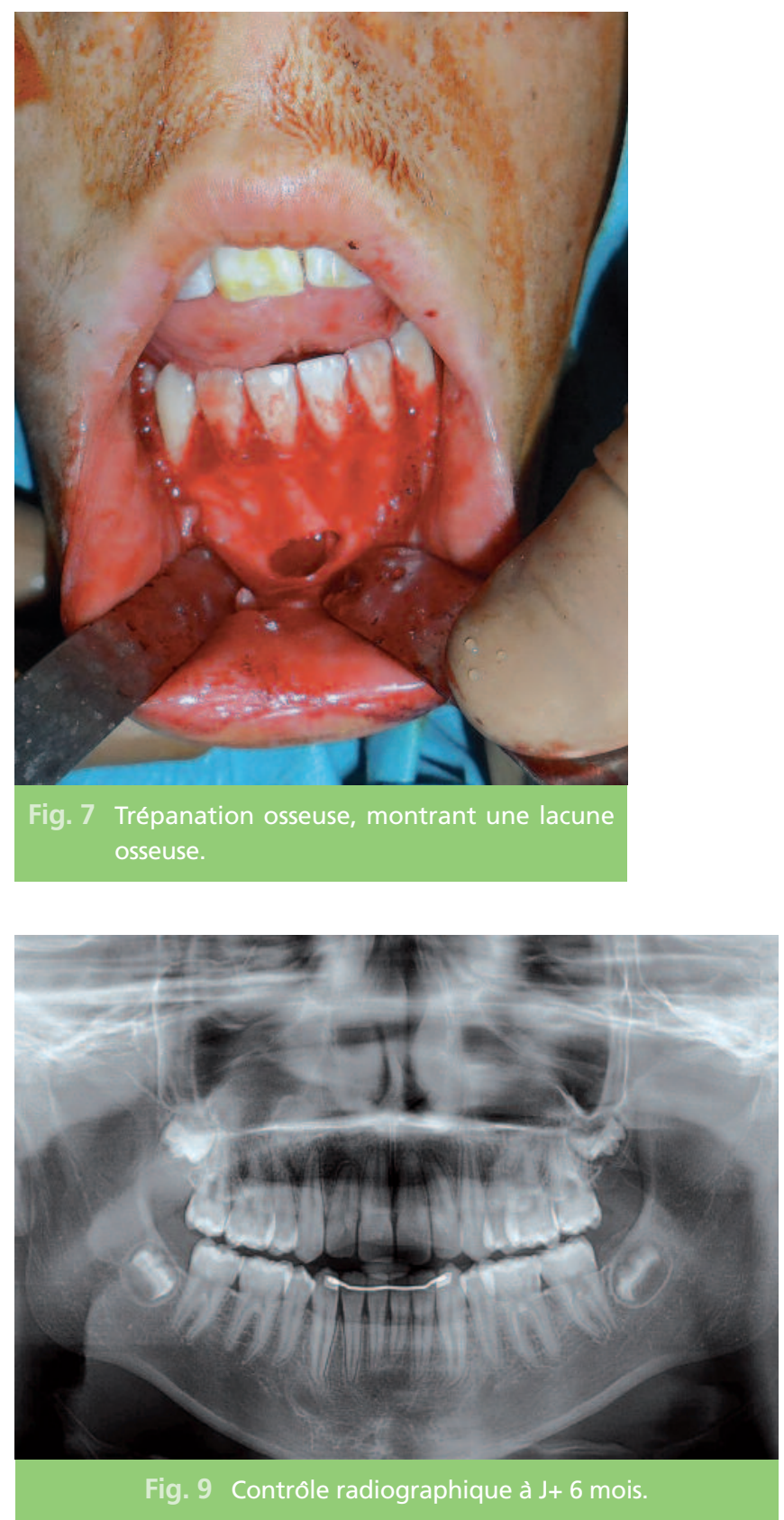


\section{Observation clinique 2}

Madame B, 67 ans, sans antécédents médicaux, est adressée pour la prise en charge d'une lésion radioclaire étendue de la mandibule (fig. 10).

II nous est impossible de retrouver d'anciennes radiographies panoramiques. La clinique est asymptomatique, l'aspect endo-buccal est normal et la palpation met en évidence une consistance osseuse.

L'aspect radiographique montre une lésion radioclaire étendue, multilobulée, cernée de zone d'ostéosclérose.

Une exérèse chirurgicale est programmée, aboutissant à la découverte d'une lacune contenant un peu de fluide sanguin. Un diagnostic préopératoire de kyste osseux solitaire est alors posé. Les suites se sont réalisées simplement.

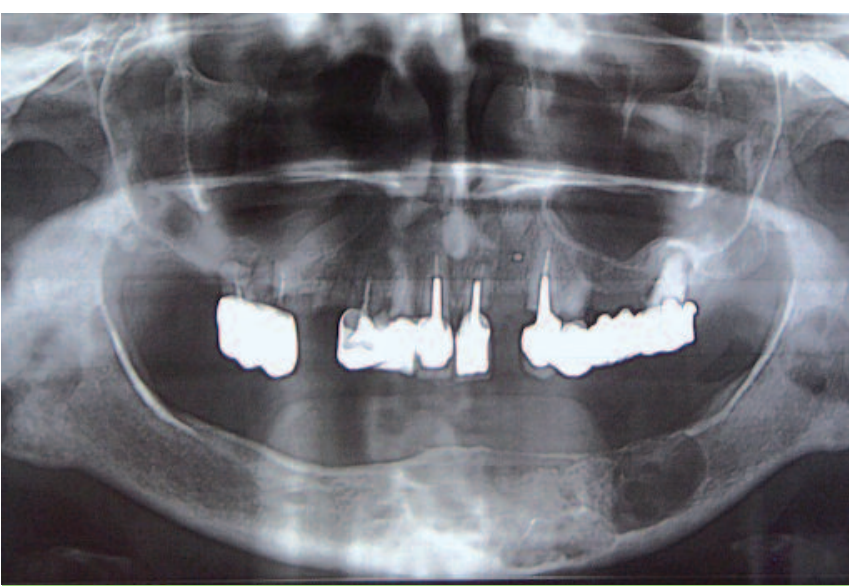

Fig. 10 Patiente âgée de 67 ans, image lacunaire plurilobulée, découverte chirurgicale d'un kyste osseux solitaire.

\section{Observation clinique 3 (fig. 11 et fig. 12)}

Monsieur D, 19 ans est adressé pour l'avulsion de la 38 enclavée.

L'examen radiographique panoramique de routine met en évidence, en plus de la 38 incluse

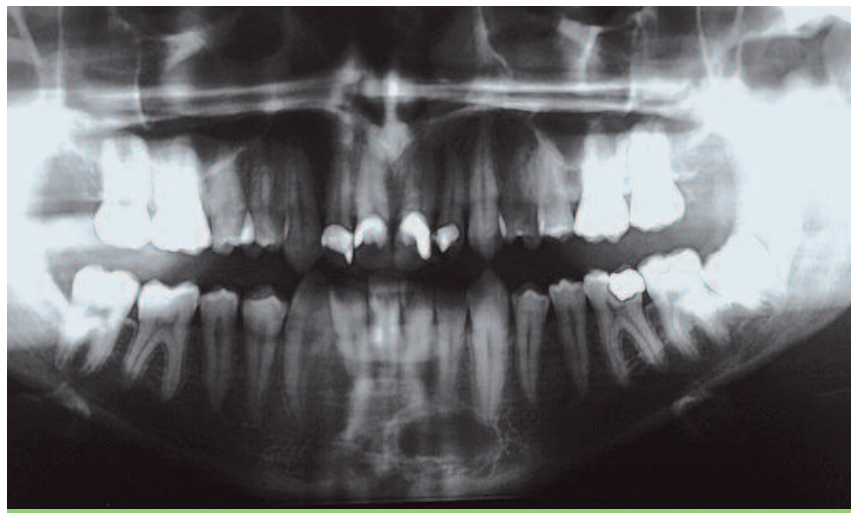

Fig. 11 Cas clinique, patient de 20 ans, image radioclaire symphysaire. une lacune radioclaire appendue à l'apex de la 33, débordant médialement.

La lacune est ceinturée d'une zone d'ostéosclérose.

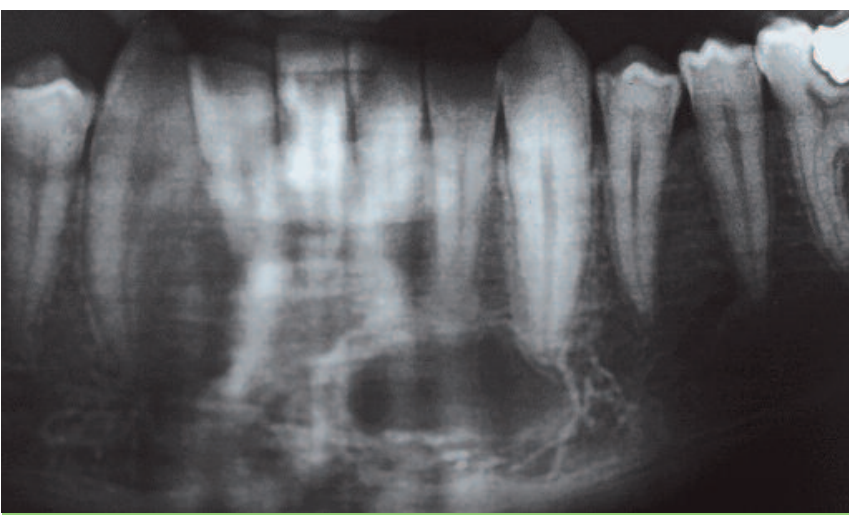

Fig. 12 Radioclareté apicale sur 33.34.35, dent répondant positivement aux tests. Découverte clinique d'un kyste osseux. 
Les tests de vitalité sur 33.32.31 ne mettent pas en évidence de nécrose pulpaire et la lésion apparaît totalement asymptomatique. Le traitement a consisté en la réalisation simultanée de la 38 incluse et un abord chirurgical de la zone lacunaire symphysaire met-

\section{Épidémiologie}

Les kystes osseux solitaires se développent avec une nette prédominance sur les métaphyses des os longs (tibia, fémur et humérus), découverts le plus souvent au détour de fractures traumatiques.

Les maxillaires et la mandibule peuvent être touchés par le développement de ces lésions, représentant environ $2 \%$ des kystes des mâchoires [2-4], touchant majoritairement la mandibule (98\%) [2-4], plus particulièrement dans sa portion antérieure [2], plus exceptionnelle aux maxillaires (2\%).

Des localisations multiples peuvent être observées dans $10 \%$ des cas [2], les localisations maxillaires étant retrouvées principalement sur ces patients à kystes osseux multiples [4].

\section{Étiopathogénie}

\section{Étiologie}

À ce jour encore controversée, l'étiologie traumatique constitue I'hypothèse initiale depuis les années 1970 [18].

D'autres étiologies, dont des dégénérescences tumorales ou des anomalies de la croissance osseuse ont été proposées depuis. tant en évidence une cavité pneumatisée. Après rinçage à la Bétadine ${ }^{\circledR}$ de la cavité, un léger curetage est effectué afin d'obtenir un saignement peropératoire dans cette lésion. Les suites ont été simples, avec un léger œdème postopératoire.

Il s'agit d'une pathologie majoritairement constatée chez le sujet jeune, aux alentours de la deuxième décade [5], plus rarement chez le sujet âgé $[4,6]$ avec une égalité de répartition homme/femme.

Toutes les zones de la mandibule peuvent être touchées, y compris le condyle, mais avec deux sites de prédilection que sont la région antérieure de la mandibule [2] et la région prémolomolaire [3].

Des localisations bilatérales sont parfois décrites [7].

Les kystes osseux solitaires sont aussi décrits dans un syndrome rare, l'incontinentia pigmenti [19], ainsi que sur des patients atteints de drépanocytose [12].

\section{Y a-t-il une implication des traitements orthodontiques?}

Une possible relation entre le développement de ces lésions et les traitements orthodontiques (22\% des cas) est prudemment évoquée par Velez [9], I'auteur n'excluant pas la possibilité 
d'un biais, alors que l'étude de Copete met en évidence que $13 \%$ des cas sont identifiés chez des patients en cours de traitement orthodontique [3].

Les relations entre le développement du kyste osseux solitaire et les traitements orthodontiques restent toutefois incertaines et à déterminer [3].

\section{Y a-t-il une implication des traumatismes chirurgicaux ?}

Dans la même veine des étiologies traumatiques, il a été décrit le développement d'un kyste osseux solitaire de l'angle de la mandibule dont l'étiologie est attribuée à l'avulsion d'une troisième molaire incluse réalisée cinq ans auparavant [10]. Là aussi, les relations entre ces deux phénomènes restent à préciser et à confirmer.

\section{Théorie traumatique}

Cette hypothèse est la plus souvent avancée, mais elle est encore très controversée car cette notion de traumatisme n'est mise en évidence que dans $7 \%$ [9] à $50 \%$ des cas [5].

Un traumatisme sur un os spongieux entouré d'os cortical entraîne la formation d'un hématome et une thrombose de l'artère terminale, entraînant une stase veineuse provoquant une nécrose de la moelle osseuse et donnant naissance à une cavité pathologique, le volume de la cavité progressant ensuite par une restriction du drainage veineux.

\section{Anomalie de la croissance osseuse}

Une anomalie du développement osseux permet d'expliquer les localisations préférentielles de ces pseudo-kystes au voisinage des cartilages de conjugaison [11].
Ces lésions se développent habituellement pendant la préadolescence ou l'adolescence, dans des zones où le remodelage osseux est très actif, faisant évoquer une relation entre ce remodelage et la lésion pseudo-kystique : une dystrophie osseuse circonscrite ou un trouble de la résorption osseuse entraînerait probablement une nécrose aseptique, secondaire à une ischémie locale.

À la mandibule, le point d'ossification primaire siège à proximité immédiate du foramen mentonnier, région anatomique fréquemment touchée par le kyste osseux solitaire. Il existe donc une probable relation entre kyste osseux solitaire et remodelage osseux.

Cette hypothèse est confortée par la fréquence élevée des kystes osseux solitaires chez les patients atteints de drépanocytose [12] (problème de synthèse de l'hémoglobine avec micro-infarctus, y compris dans l'os).

\section{Dégénérescence de lésion tumorale bénigne}

Plusieurs hypothèses évoquent une transformation tumorale au cours du développement de lésion fibro-osseuse $[2,9,13-15]$, à partir de lésion de dysplasies fibreuses [16] ou d'infections à bas grade [10].

L'association entre kyste osseux solitaire et les lésions fibro-osseuses (plus particulièrement les dysplasies périapicales florides) semble toucher les patients plus âgés [13] et pourrait s'expliquer par un découplage de l'activité ostéoblastique et ostéoclastique [13].

\section{Pathogénie}

À ce jour, la pathogénie des kystes osseux est toujours inconnue mais un consensus se dessine : une altération du système vasculaire entraîne une ischémie locale post-hémorragique 
à l'origine d'une nécrose aseptique et l'apparition d'un transsudat dans la cavité favorisant la résorption osseuse [17].

\section{Clinique}

Le kyste osseux solitaire des maxillaires se présente le plus souvent de manière asymptomatique, de découverte fortuite au cours de radiographie de routine [3].

La sémiologie est le plus souvent inexistante, quelques fois fruste et peu spécifique.

Quelques cas de douleurs et de tuméfactions ont été décrits $[3,7]$.

\section{Radiographie}

L'image radiographique va varier en fonction du degré d'extension de la lésion.

\section{Radioclareté de petite étendue}

On observe une ostéolyse bien limitée [9], le plus souvent uniloculaire, ceinturée par une bordure osseuse.

Cette bordure osseuse présente dans $50 \%$ des cas une sclérose périphérique [3] parfois associée à une atteinte partielle de la corticale.

Les limites de la lésion forment des images typiques de festons entre les apex des dents adjacentes, cela dans 50 [13] à $70 \%$ des cas [3] avec un aspect festonné (scalloping des Anglo-Saxons) contournant les éléments radiculaires.

Cet aspect festonné constituerait un signe pathognomonique du kyste osseux solitaire [13].
L'extension du kyste serait ensuite favorisée par une augmentation de la pression intramédulaire.

Les tests de vitalité des dents adjacentes sont positifs [6], des signes de paresthésies étant décrits dans moins de $3 \%$ des cas [18].

Les kystes osseux peuvent aussi être associés à des lésions fibro-osseuses $[4,13,14,16]$, cellesci étant liées au remplacement d'un tissu osseux par un tissu fibreux, contenant des proportions variables de calcifications.

Il n'y a pas de résorption radiculaire associée [4, 9] et les lamina dura peuvent persister, cette caractéristique radiographique étant retrouvée dans $34 \%$ des cas, associée aux festons interradiculaires [4].

La lésion peut apparaître comme multilobulaire, du fait de la présence de mince pseudoseptums mais se localise généralement de manière unilatérale (75 \% des cas).

L'intérieur de la lésion présente plusieurs caractéristiques : présence de trabécules, radioclareté complète, apparence " nuageuse ».

Dans les cas de figures associés aux lésions fibroosseuses, on retrouve en plus des hypercémentoses autour des apex dentaires [4].

\section{Radioclareté de grande étendue}

Avec l'accroissement de la taille de la lésion, I'aspect radiographique devient très polymorphe, 
franchement polygéodique, évoquant des lésions tumorales de types kératokystes ou améloblastomes (fig. 8).

Le volume des lésions peut devenir considérable [7], parfois bilatéral et entraînant une dispari-

\section{Histologie}

L'examen histologique est difficile de par l'absence de matériel récupérable.

La paroi interne de la cavité apparaît tapissée d'une mince couche conjonctive, avec une absence de paroi épithéliale.

Le contenu de la cavité est variable, la plupart du temps pneumatisée (surtout dans sa localisation mandibulaire), parfois remplie d'un fluide sanguin, séreux ou séro-hématique. tion partielle ou totale du canal mandibulaire dans la lésion [4].

Les examens TDM montrent des corticales très amincies mais sans solution de continuité [17].

Le contenu de la cavité semble lié à la durée d'évolution de la lésion, une période d'évolution longue permettant une résorption progressive du fluide intrakystique [20], aboutissant à des cavités déshabitées.

Sur les patients beaucoup plus âgés, il peut apparaître du tissu de granulation, contenant des granules de cholestérol et des cellules géantes multinucléaires [4].

\section{Diagnostic différentiel}

La pose d'un diagnostic différentiel de certitude nécessitera le plus souvent une exploration chirurgicale, l'extension de la lésion pouvant donner des images très polymorphes, ouvrant des hypothèses diagnostiques larges.

\section{Devant des lésions de tailles réduites}

Il faut évoquer :

\section{> les kystes radiculo-dentaires apicaux et latéraux ou résiduels (fig. 13)}

Développées à partir de nécroses pulpaires, ces lésions contiennent des résidus épithéliaux et provoquent la formation et I'accroissement d'un kyste par hyperpression osmotique. Les kystes radiculo-dentaires apicaux et latéraux ne diffèrent que par leur topographie.

- Signes radiographiques : ostéolyse péri-apicale, appendue à l'apex d'une dent, prenant

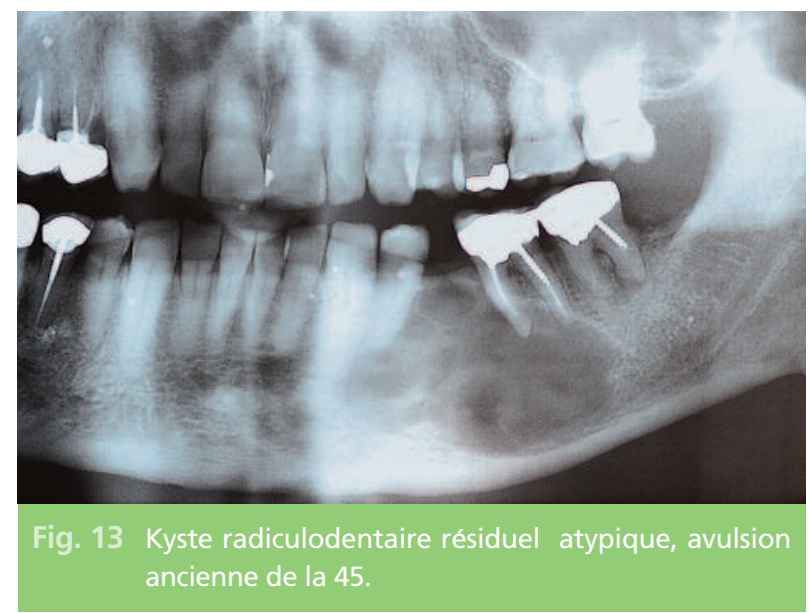


parfois des volumes importants, soufflant les corticales et pouvant évoquer des kératokystes.

- Anatomopathologie : la lésion contient des cristaux de cholestérol, au milieu d'un liquide visqueux, limité par une membrane épithéliale, d'épaisseur variable ;

\section{> un kyste anévrismal osseux}

Pseudo-kyste rempli de sang, parfois à l'extérieur du tissu osseux, caractérisé par une symptomatologie franche, avec tuméfaction, douleurs, déplacement ou lésions des dents avoisinantes. Ces lésions sont assimilées à des processus ostéodystrophiques réactionnels, d'étiopathogénie incertaine et qui pourrait former un phénomène de réparation secondaire à une anomalie vasculaire du tissu osseux.

- Signes radiographiques : dans les formes de petite taille, on retrouve une lésion monogéodique bien définie, évoluant dans les formes plus volumineuses vers des lésions multilobulaires en « bulle de savon».

Des aspects radio-opaques et des lésions mixtes radioclaires/radiodenses peuvent être retrouvés occasionnellement.

- Anatomopathologie : dans leur forme classique, il s'agit de lacunes remplies de sang, riches en fibroblaste et en cellules géantes, associées ou non à des formations d'os.

Une forme " solide » existe, ou la composante vasculaire est limité mais où prédomine une prolifération fibroblastique ;

\section{> des lésions fibro-osseuses [16]}

dont les dysplasies péri-apicales aiguës [8] et les dysplasies orales florides [14].

- Dysplasie péri-apicale aiguë : lésion le plus souvent asymptomatique, caractérisée par une image radioclaire, parfois mixte radioclaire/radiodense, en étroite relation avec les apex dentaires.
- Dysplasie orale floride : là aussi liée aux portions dentées des maxillaires, avec des images radioclaires ou mixtes identiques à la dysplasie péri-apicale aiguë mais touchant les quatre quadrants des maxillaires et prédominant chez les femmes noires.

- Anatomopathologie : proche histologiquement des fibromes cémento-ossifiant, où le tissu conjonctif prédomine, richement vascularisé mais avec de petites zones de travées osseuses dispersées.

\section{Devant des lésions de tailles plus importantes}

Il faut évoquer :

\section{$>$ des granulomes à cellules géantes}

Ils sont aussi nommés granulomes réparateurs à cellules géantes. D'étiologie incertaine, considérée comme une lésion réactionelle d'évolution lente, bien que des formes agressives ou récidivantes existent.

- Signes radiographiques : très varié, lithique et uniloculaire dans les formes de petite taille, simulant fréquemment un kyste odontogénique pour prendre des aspects multiloculaires dans les grandes lésions, avec de minces cloisons osseuses.

- Anatomopathologie : aspect très variable, des groupes de cellules géantes multinucléés séparées par des zones de fibroblastes sont considérés caractéristiques du granulome à cellules géantes ;

\section{$>$ des fibromes odontogéniques}

Tumeur bénigne issue des fibroblastes pulpaire ou du ligament parodontal.

- Signes radiographiques : lésion radioclaire uni- ou polygéodique, refoulant les corticales, bien limitée, avec ou sans rhizalyse, prédominant au maxillaire. 
- Anatomopathologie : lésion fibroblastique, formée de bandes de fibroblaste entrelacé et séparées par des zones moins cellulaires;

\section{> des fibromes amélobastiques}

Tumeur mixte formée d'éléments épithéliaux et mésenchymateux, ne contenant pas de tissus minéralisés odontogènes et prédominant à la mandibule.

- Signes radiographiques : lésion radioclaire bien limitée, uni- voire plurilobulaire lorsque la lésion devient plus volumineuse et fréquemment associée à une dent incluse (évoquant un kyste folliculaire).

- Anatomopathologie : caractérisé par une double composante, épithéliale et conjonctive, avec des ilots épithéliaux rappelant l'organe de l'émail et du tissu conjonctif évoquant la papille dentaire ;

\section{> des kystes épidermoïdes}

Caractérisés malgré leur caractère bénin par une croissance plus agressive et une forte tendance à la récidive.

- Signes radiographiques : débute par une image radioclaire géodique, souvent volumi-

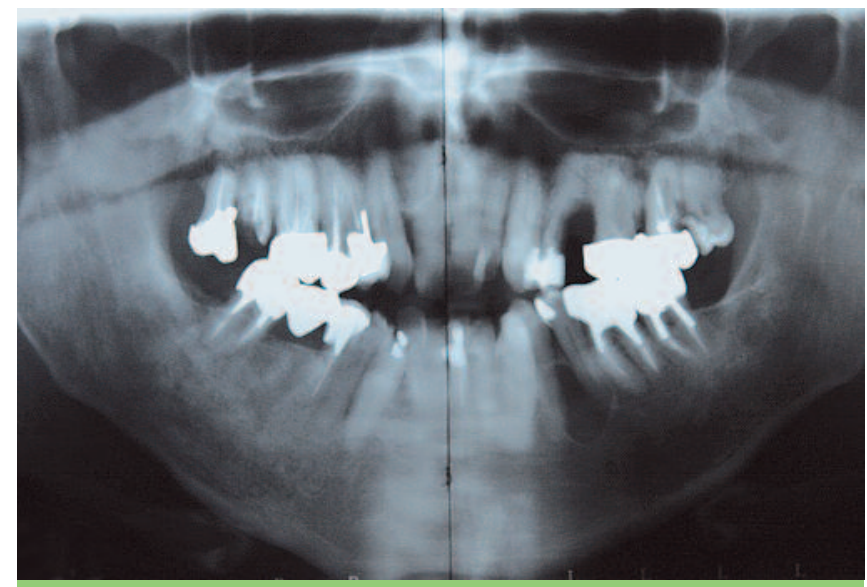

Fig. 14 Kyste épidermoide de la région de 33.34. neuse, bien limitée, évoluant vers des lésions festonnées, polygéodiques.

Les kératokystes peuvent aussi prendre un aspect en forme de poire (fig. 14) s'insinuant entre les racines dentaires, parfois associé à des rhizalyses dentaires.

- Anatomopathologie : tumeur bordée d'un épithélium malpighien, avec en périphérie une coque conjonctive contenant des ilots épithéliaux ;

\section{> des améloblastomes (fig. 15)}

Issus de résidus de l'épithélium odontogénique, cette lésion se caractérise par son agressivité locale.

Il s'agit d'une tumeur à topographie majoritairement mandibulaire, se caractérisant cliniquement le plus souvent par sa découverte fortuite, parfois par une voussure osseuse.

- Signes radiographiques : lésion bien limitée, volumineuse, multigéodique en bulle de savon, refoulant les corticales et entraînant fréquemment des rhizalyses.

- Anatomopathologie : on retrouve des ilots d'épithélium, de taille variable, associés à un stroma conjonctif de collagène, en quantité variable.

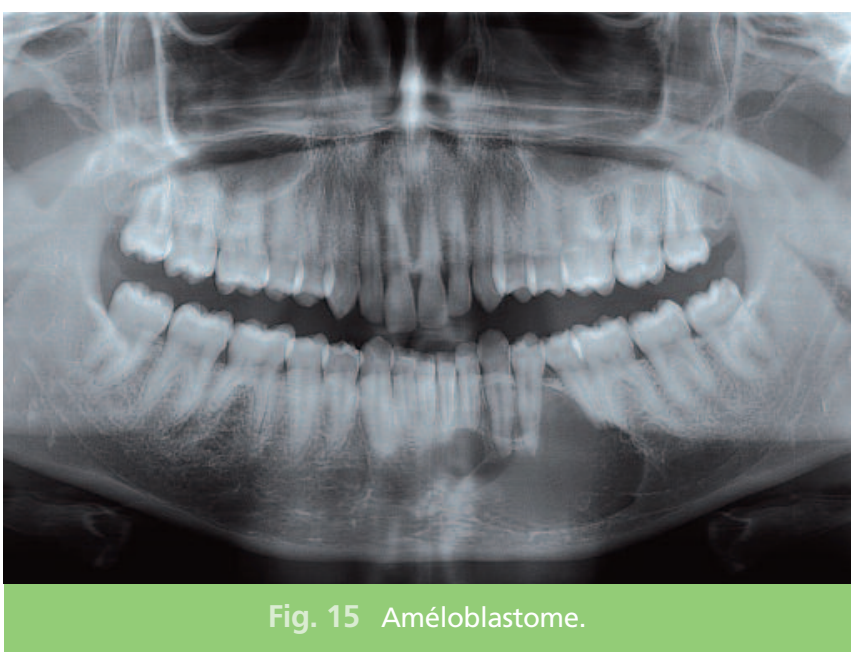




\section{Thérapeutique}

La trépanation chirurgicale constitue le traitement de choix, d'abord pour confirmer le diagnostic puis secondairement pour promouvoir la cicatrisation par l'ouverture de la lésion permettant la formation d'un caillot sanguin et une réossification [4]. II peut être possible de différer l'intervention lorsque les dents présentent une édification radiculaire incomplète dans la zone de la lésion [4]. Un échec thérapeutique a été signalé avec cette technique, qui nécessitera

\section{Conclusion}

Le kyste osseux solitaire constitue une pseudotumeur bénigne, de topographie multiple, pouvant intéresser les maxillaires et la mandibule.

Des formes de grandes étendues existent, pouvant poser d'importants doutes diagnostiques en particulier chez le patient âgé. L'étiologie de ces lésions reste à préciser, en particulier avec secondairement l'injection de sang autogène après une deuxième intervention [21].

Un certain nombre d'auteurs ont rapporté des cas de rémissions spontanées, chez des patients de moins de 20 ans, avec un aspect radiographique de l'os plus dense que celui du voisinage $[6,4,10]$ faisant proposer à certains, en l'absence de symptomatologie et chez des patients jeunes, d'attendre une éventuelle guérison spontanée [22]. de possibles étiologies iatrogènes (orthodontie et avulsions dentaires).

Le traitement reste simple, dominé par la trépanation chirurgicale bien que des abstentions thérapeutiques aient pu entraîner des guérisons spontanées et qu'un cas particulier ait nécessité une réintervention et une injection de sang autologue.

\section{Bibliographie}

1. Kramer IRH, Pindborg JJ, Shear M. Histological typing of odontogenic tumours. $2^{\text {nd }}$ ed. Berlin: Springer, 1992.

2. Kaugars GE, Cale AE. Traumatic bone cyst. Oral Surg Oral Med Oral Pathol 1987;63(3):318-24.

3. Copete MA, Kawamata A, Langlais RP. Solitary bone cyst of the jaws: radiographic rewiew of 44 cases.

Oral Surg Oral Med Oral

Pathol Radiol Endod

1998;85(2):221-5.

4. Saito $Y$, Hoshina $Y$, Nagamine T, Nakajima T, et al.

Simple bone cyst. A clinical and histopathologic study of fifteen cases

Oral Surg Oral Med Oral Pathol 1992;74(4):487-91.

5. Howe GL. 'Haemorraghic cysts' of the mandibule. I. II. Br J Oral Surg 1965;3(1-2):55-91.

6. Cowan CG. Traumatic bone cysts of the jaws and their presentation. Int J Oral Surg 1980;9(4):287-91.

7. Patrikiou $A$, Sepheriadou-Mavropoulou T, Zambelis G. Bilateral traumatic bone cyst of the mandibule.

A case report. 
Oral Surg Oral Med Oral Pathol 1981;51(2):131-3.

8. Harris SJ, OCarroll MK, Gordy FM.

Idiopathic bone cavity (traumatic bone cyst) with the radiographic appearance of a fibro-osseous lesion. Oral Surg Oral Med Oral Pathol 1992;74(1):118-23.

9. Velez I, Siegel MA, Mintz SM, Rolle R.

The relationship between idiopathic bone cavity and orthodontic tooth movement: analysis of $\mathbf{4 4}$ cases. Dentomaxillofac Radiol 2010;39(3):162-6.

10. Pogrel MA.

A solitary bone cyst possibly caused by removal of an impacted third molar. J Oral Maxillofac Surg 1987;45(8):721-3.

11. Ludwig A, Merten HA.

Kyste osseux traumatiques: pathogenèse, clinique et thérapie.

Rev Mens Suisse

Odontostomatol

1995;105(1):63-70.

12. Noujaim $S$, Alantar ALP, Galeazi JM, Genet P, Maman L. Kyste osseux solitaire chez un enfant atteint de drépanocytose.

Med Buccale Chir Buccale 2003;9:105-1.

13. Chadwick JW, Alsufyani NA, Lam EW.

Clinical and radiographic

features of solitary and cemento-osseous dysplasia-associated simple bone cysts.

Dentomaxillofac Radiol 2011;40(4):230-5.

14. Melrose RJ, Abrams AM, Mills BG.

Florid osseous dysplasia. A clinical-pathologic study of thirty-four cases. Oral Surg Oral Med Oral Pathol 1976;41(1):62-82.

15. Moule I.

Unilateral multiples solitary bone cysts.

J Oral Maxillofacial Surg 1988;46(4):320-3.

16. Harra H, Ohishi M, Higuchi Y. Fibrous dysplasia of the mandibule associated with large solitary bone cyst. J Oral Maxillofac Surg 1990;48(1):88-91.

17. Harnet JC, Feki A, Lombardi T. Kyste osseux solitaire des maxillaires caractéristiques radiologiques et déterminisme du processus pathogène.
Med Buccale Chir Buccale 2010;16:95-100.

18. Heubner GR, Turlington EG. So-called traumatic (hemorrhagic) bone cysts of the jaws. Review of the literature and report of two unusual cases. Oral Surg Oral Med Oral Pathol 1971;31(3):354-65.

19. Baddour HM, Steed DL, Tilson HB.

Incontinentia pigmenti: report of case. J Oral Surg 1981;39(1):57-9.

20. Xanthinaki AA, Choupis KI, Tosios K, Pagkalos VA, Papanikolaou SI. Traumatic bone cyst of the mandibule of possible iatrognic origin: a case report and brief review of the literature. Head Face Med 2006;2:40.

21. Precious DS, McFadden LR. Treatment of traumatic bone cyst of mandibule by injection of autogeneic blood. Oral Surg Oral Med Oral Pathol 1984;58(2):137-40.

22. Naumann S, Filippi $A$. Les pseudokystes du maxillaire inférieur. Rev Mens Suisse Odontostomatol 2002;112(10):1026-30.

\section{SUMMARY}

\section{Solitary bone cyst of the mandibule. Diagnosis, evolution and treatment}

Christian MILIN

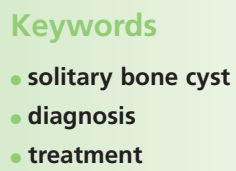

The identification of a solitary bone cyst of the mandibule, concerning a teenager, allowed us to follow it's evolution during 4 years.

After a review of a different suspected etiology and pathogenesis, we are able to expose the diagnosis and the treatment of this clinical case. We also present two cases of a bigger solitary bone cyst, one of a bigger solitary bone cyst to an old lady. 